\title{
The contribution of the toxicity of a glycerol-egg yolk-citrate cryopreservative to the decline in human sperm motility during cryopreservation
}

\author{
E. A. McLaughlin, W. C. L. Ford and M. G. R. Hull \\ University Department of Obstetrics and Gynaecology, St Michael's Hospital, Southwell Street, \\ Bristol BS2 8EG, UK
}

\begin{abstract}
Summary. The contribution of the toxicity of glycerol-egg yolk-citrate (GEYC) cryopreservative medium to the loss of function of human spermatozoa during cryopreservation was determined by investigating the effect of mixing semen with the medium on sperm motility. The percentage of progressively motile spermatozoa, velocity $\left(\mu \mathrm{m} \mathrm{s}^{-1}\right)$ and lateral head displacement $(\mu \mathrm{m})$ (mean $\pm \mathrm{SEM}, n=28)$ were $55 \pm 4 \cdot 1,47 \pm 2 \cdot 7$, $4.4 \pm 0.2$ and $32 \pm 3 \cdot 8,40 \pm 2 \cdot 5,3 \cdot 6 \pm 0.25$ and $15 \pm 2 \cdot 5,28 \pm 1 \cdot 1,2 \cdot 8 \pm 0 \cdot 15$ in suspensions of washed spermatozoa prepared from fresh, GEYC-treated and frozenthawed semen, respectively. The variables changed only slightly after incubation for $3 \mathrm{~h}$. The toxicity of GEYC did not vary significantly between samples which survived the complete freeze-thaw cycle well or very poorly. The toxicity of GEYC is responsible for about $50 \%$ of the loss of progressively motile spermatozoa during the complete cryopreservation process, but has little effect on the quality of motility. Susceptibility to GEYC does not explain observed differences in the ability of semen samples to survive freezing.
\end{abstract}

Key'ords: cryopreservation; spermatozoa; motility; glycerol; donor insemination; human

\section{Introduction}

Male infertility is the commonest single cause of childlessness (Hull et al., 1985), and donor insemination (DI) is frequently the only way in which affected couples can have children. To prevent the spread of human immunodeficiency virus (HIV), frozen semen is always used for DI. This has numerous practical advantages but it is generally accepted that frozen semen is less fertile than fresh semen (see Watson, 1990) and only a few men produce semen that survives freezing and thawing to a satisfactory extent. Consequently there is a need to develop better cryopreservation techniques for human spermatozoa.

Cell damage during freezing occurs by two principal mechanisms. If cooling is too fast to permit excess water to diffuse out of the cell, intracellular ice forms. However, if cooling is too slow, the cells are exposed to high intracellular solute concentrations as bulk water freezes (solution effects). Successful cryopreservation requires a freezing rate that is an optimal compromise between these two processes. The addition of cryoprotectants increases the proportion of unfrozen water at a given temperature and so decreases the concentration of electrolytes and diminishes the impact of solution effects. This increases the range of cooling rates over which cells can survive and the addition of cryoprotectants is essential for the successful cryopreservation of many types of cell including spermatozoa. Glycerol is one of the most successful cryoprotective agents discovered so far (see Mazur, 1984; Watson, 1990). Unfortunately cryopreservatives, including glycerol, are in themselves toxic to spermatozoa. This is due in part to the osmotic stress that they impose on the cell but glycerol may have direct effects on the structure of the plasma membrane and on the metabolism of the cell (see Hammerstedt et al., 1990). 
After the cryopreservation of human semen, there is a decrease in the proportion of spermatozoa that are motile and of those that have intact plasma membranes as well as a decrease in the average velocity of those spermatozoa that remain motile. However the extents of these three changes are not correlated (McLaughlin et al., 1992). A considerable proportion of the damage may be due to the toxicity of cryopreservatives that have a high glycerol content (Critser et al., 1988). We investigated the contribution of the toxicity of the glycerol-egg yolk-citrate cryopreservative used in our laboratory to the deterioration of sperm motility observed after freezing and thawing.

\section{Materials and Methods}

\section{Semen preparation and experimental design}

Semen was collected by masturbation from a group of 18 donors whose semen exhibited a wide range of cryosurvival characteristics. The semen was allowed to liquefy and was divided into three parts. The first portion $(200 \mu l)$ was washed three times by centrifugation in Biggers, Whitten and Whittingham medium containing $1 \% \mathrm{w} / \mathrm{v}$ bovine serum albumin (BWW) (Aitken, 1983) and the final pellet was resuspended in $200 \mu \mathrm{l}$ BWW medium and a $5 \mu \mathrm{l}$ sample was taken for motility analysis. The ability of the spermatozoa to sustain their motility was examined by incubating the remainder of the suspension for $3 \mathrm{~h}$ at $37^{\circ} \mathrm{C}$ under $95 \%$ air: $5 \% \mathrm{CO}_{2}$ and then removing a second sample for measurement. The second portion $(200 \mu \mathrm{l})$ was mixed with an equal volume of glycerol-egg yolk-citrate (GEYC)

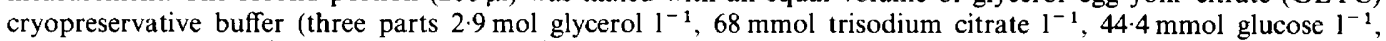
$44.4 \mathrm{mmol}$ fructose $\mathrm{l}^{-1}, 26 \cdot 7 \mathrm{mmol}$ glycine $\mathrm{l}^{-1}, \mathrm{pH} 7 \cdot 2-7 \cdot 4$ mixed with one part fresh egg yolk) to give a final glycerol concentration of $1.09 \mathrm{moll}^{-1}$ (McLaughlin et al., 1990) and allowed to stand at room temperature for 20 min before dropwise dilution with $1.8 \mathrm{ml}$ BWW medium over $5 \mathrm{~min}$, washing, resuspension, incubation and motility assessment as described above. A 20 min exposure was chosen because it corresponds to the average period that semen intended for donor insemination is in contact with GEYC before freezing in this laboratory. The remainder of the ejaculate was mixed with an equal volume of GEYC loaded into $0.25 \mathrm{ml}$ plastic straws and frozen in a Nicool LM-10 semiprogrammable freezer (McLaughlin et al., 1990) before plunging into liquid nitrogen. Straws were removed from the liquid nitrogen for analysis and allowed to thaw at room temperature for $10 \mathrm{~min}$ before the semen was gradually diluted with BWW medium, washed, resuspended and incubated for motility assessment as described above.

\section{Motility analysis}

Sperm motility parameters were measured with a Hamilton Thorn Motility Analyser Version 7 (Hamilton Thorn Research Inc., 30A Cherry Hill Drive, Danvers, USA). A $10 \mu \mathrm{l}$ sample of the sperm suspension was placed on a Makler counting chamber (Sefi-Medical Instruments, Haifa, Israel) and the glass coverslip pressed down firmly. Motility was measured using the internal microscope of the analyser with the instrument settings as follows. Analyser set up - temperature: $37^{\circ} \mathrm{C}$; diluent: sample: 0:1; chamber: Makler, $(10 \mu \mathrm{m})$; image type: phase contrast; field selection: select; calculate ALH: Yes; Morphology: Live; Beat frequency: Yes; Automatic Sort: No; Allow Static Override: No. Main Gates; Frames: 20; Frame rate: $25 \mathrm{~s}^{-1}$; Minimum contrast: 9; Minimum size: 8 ; LO/HI size gates: $0.51 \cdot 3$; LO/HI intensity gates: 0.5 1.5; Non-motile head size: 14 ; Non-motile intensity: 210 ; Medium VAP value: 25 ; Low VAP value: 10; Low cells motile: No. Theshold STR: 80 . At least 200 spermatozoa were analysed for each sample and measurements were made as soon as possible after the chamber had achieved the correct temperature to prevent errors due to evaporation.

\section{Statistical analysis}

The results were analysed by a multiway analysis of variance. When this demonstrated a significant effect, differences between individual cells or treatments were tested with the Tukey test or by paired $t$ tests or individual one-way analysis of variance.

\section{Results}

The number of progressively motile spermatozoa was reduced by $42 \%$ after mixing with GEYC buffer and by a further $53 \%$ after freezing and thawing giving a $73 \%$ reduction overall (Table 1 ).

The ejaculates were grouped according to the percentage of progressively motile spermatozoa to survive freezing and thawing before dilution in the same way that ejaculates to be used in clinical 
Table 1. Effect of mixing with glycerol-egg yolk-citrate cryopreservative (GEYC) and of subsequent freezing and thawing on the percentage of progressively motile human spermatozoa present after washing and suspension in Biggers, Whitten and Whittingham (BWW) medium in human ejaculates that survived cryopreservation to different extents

\begin{tabular}{lcccc}
\hline & \multirow{3}{*}{$\begin{array}{c}\text { Incubation } \\
\text { time }\end{array}$} & \multicolumn{4}{c}{$\%$ Motile } \\
\cline { 3 - 5 } $\begin{array}{l}\text { Survival of freezing } \\
\text { (No. of observations) }\end{array}$ & $(\mathrm{h})$ & Fresh & GEYC-treated & Frozen-thawed \\
\hline Poor $(<40)$ & 0 & $56 \pm 4 \cdot 8$ & $30 \pm 5 \cdot 1^{* * *}$ & $9 \pm 1 \cdot 4^{* * * a}$ \\
$(17)$ & 3 & $58 \pm 5 \cdot 1$ & $35 \pm 5 \cdot 1^{* *}$ & $11 \pm 1 \cdot 7^{* * *}$ \\
Borderline $(40-60)$ & 0 & $47 \pm 9 \cdot 4$ & $31 \pm 4 \cdot 8$ & $16 \pm 2 \cdot 9^{* * \mathrm{~b}}$ \\
$(6)$ & 3 & $50 \pm 4 \cdot 9$ & $31 \pm 5 \cdot 7^{*}$ & $20 \pm 4 \cdot 1^{* * *}$ \\
Good $(>60)$ & 0 & $58 \pm 13 \cdot 2$ & $43 \pm 10 \cdot 9$ & $36 \pm 7 \cdot 5^{\mathrm{c}}$ \\
$(5)$ & 3 & $60 \pm 10 \cdot 6$ & $45 \pm 9 \cdot 0$ & $27 \pm 10 \cdot 5$ \\
All & 0 & $55 \pm 4 \cdot 1$ & $32 \pm 3 \cdot 8^{* * *}$ & $15 \pm 2 \cdot 5^{* * *}$ \\
$(28)$ & 3 & $57 \pm 3 \cdot 7$ & $36 \pm 3 \cdot 7^{* * *}$ & $16 \pm 2 \cdot 5^{* * *}$ \\
\hline
\end{tabular}

Data are means \pm SEM. $\dagger(\%$ motile after freezing and thawing $/ \%$ motile when fresh $) \times 100$ (before washing). Significant differences between fresh and GEYC-treated or between GEYCtreated and frozen-thawed spermatozoa after washing. ${ }^{* * *} P<0.001, \quad{ }^{* *} P<0.01$, ${ }^{*} P<0.05$. Values with different superscripts $(\mathrm{a}, \mathrm{b}, \mathrm{c})$ are significantly different $P<0.05$ between poor, borderline and good survival groups. The presence of significant variation was detected by a three-way analysis of variance and differences between individual cells of the table were tested subsequently by one-way analysis of variance or paired $t$ tests as appropriate.

donor insemination are classified. The groups were 'good', 'borderline' and 'poor' and the percentage of progressively motile spermatozoa to survive freezing and thawing in each were $>60,40-60$ and $<40$, respectively. Significant differences between groups in line with their definition remained after dilution. However, after exposure to GEYC without freezing, survival was not significantly better in the 'good' than in the 'borderline' or 'poor' groups so that the deleterious effect of GEYC was independent of the ability of the spermatozoa to survive the complete freeze-thaw cycle (Table 1). With the possible exception of frozen-thawed spermatozoa from ejaculates that had 'good' freeze survival, the spermatozoa sustained their motility during the $3 \mathrm{~h}$ incubation and no significant effect of incubation time on the percentage of motile spermatozoa was detected in the complete data set (Table 1).

The velocity of the motile spermatozoa was significantly decreased by freezing and thawing, but the extent of the change was the same no matter what proportion of motile spermatozoa survived the process. Velocity increased slightly during the $3 \mathrm{~h}$ incubation (Table 2). Simply mixing the spermatozoa with GEYC appeared to decrease the average velocity of the spermatozoa but this was not statistically significant until after incubation for $3 \mathrm{~h}$ (Table 2).

The lateral head displacement of the spermatozoa was significantly decreased by freezing and thawing. This was true in all the survival categories but was most pronounced in the samples with poor survival. Mixing with GEYC caused a smaller decrease in lateral head displacement and this was statistically significant only in the 'poor' survival group. The lateral head displacement increased slightly during the $3 \mathrm{~h}$ incubation in all treatments and groups (Table 3).

\section{Discussion}

These data clearly confirm that cryopreservatives can be toxic towards human spermatozoa. This toxicity was responsible for at least half of the decrease in the percentage of progressively motile spermatozoa during cryopreservation in the present experiments.

Compared with the complete freeze-thaw cycle, mixing with GEYC had less effect on the quality of motility, as expressed by progressive velocity and lateral head displacement. The 
Table 2. Effect of mixing with glycerol-egg yolk-citrate cryopreservative and subsequent freezing and thawing on the progressive velocity of human spermatozoa after washing and suspension in Biggers, Whitten and Whittingham (BWW) medium in human ejaculates that survived cryopreservation to different extents

\begin{tabular}{lcccc}
\hline & \multirow{3}{*}{$\begin{array}{c}\text { Incubation } \\
\text { time }\end{array}$} & \multicolumn{3}{c}{ Velocity $\left(\mu \mathrm{m} \mathrm{s}^{-1}\right)$} \\
\cline { 3 - 5 } (No. of observations) & $(\mathrm{h})$ & Fresh & GEYC-treated & Frozen-thawed \\
\hline Poor $(<40)$ & 0 & $48 \pm 3 \cdot 8$ & $39 \pm 3 \cdot 2$ & $29 \pm 1 \cdot 5^{* * *}$ \\
$(17)$ & 3 & $51 \pm 3 \cdot 1$ & $40 \pm 3 \cdot 7^{*}$ & $29 \pm 1 \cdot 8^{* * *}$ \\
Borderline $(40-60)$ & 0 & $49 \pm 6 \cdot 4$ & $43 \pm 7 \cdot 5$ & $25 \pm 1 \cdot 7^{* *}$ \\
$(6)$ & 3 & $52 \pm 6 \cdot 2$ & $49 \pm 7 \cdot 3$ & $35 \pm 2 \cdot 8^{*}$ \\
Good $(>60)$ & 0 & $44 \pm 3 \cdot 9$ & $37 \pm 2 \cdot 8$ & $27 \pm 3 \cdot 1^{*}$ \\
$(5)$ & 3 & $50 \pm 6 \cdot 5$ & $44 \pm 4 \cdot 7$ & $36 \pm 2 \cdot 6$ \\
All & 0 & $47 \pm 2 \cdot 7$ & $40 \pm 2 \cdot 5$ & $28 \pm 1 \cdot 1^{* * *}$ \\
$(28)$ & 3 & $51 \pm 2 \cdot 5$ & $43 \pm 2 \cdot 8^{* *}$ & $31 \pm 1 \cdot 4^{* * *}$ \\
\hline
\end{tabular}

Data are means \pm SEM. $+(\%$ motile after freezing and thawing $/ \%$ motile when fresh $) \times 100$ (before washing). Significant differences between fresh and GEYC-treated or between GEYCtreated and frozen-thawed spermatozoa after washing. $* * * P<0.001, \quad * * P<0.01$, ${ }^{*} P<0.05$. The presence of significant variation was detected with a three-way analysis of variance and differences between individual cells of the table were tested subsequently by paired $t$ tests.

Table 3. The effect of mixing with glycerol-egg yolk-citrate cryopreservative and of subsequent freezing and thawing on the lateral head displacement of spermatozoa after washing and suspension in Biggers, Whitten and Whittingham (BWW) medium in human ejaculates that survived cryopreservation to different extents

\begin{tabular}{|c|c|c|c|c|}
\hline \multirow{2}{*}{$\begin{array}{l}\text { Survival of freezing } \dagger \\
\text { (No. of observations) }\end{array}$} & \multirow{2}{*}{$\begin{array}{l}\text { Incubation } \\
\text { time } \\
\text { (h) }\end{array}$} & \multicolumn{3}{|c|}{ Lateral head displacement $(\mu \mathrm{m})$} \\
\hline & & Fresh & GEYC-treated & Frozen-thawed \\
\hline Poor $(<40)$ & 0 & $4 \cdot 1 \pm 0 \cdot 35$ & $3 \cdot 2 \pm 0 \cdot 24^{*}$ & $2 \cdot 7 \pm 0 \cdot 20^{* * *}$ \\
\hline (17) & 3 & $4.5 \pm 0.32$ & $3 \cdot 9 \pm 0.31$ & $2 \cdot 8 \pm 0.21 * * *$ \\
\hline Borderline $(40-60)$ & 0 & $5 \cdot 0 \pm 0.60$ & $4 \cdot 1 \pm 0.79$ & $2 \cdot 9 \pm 0.37^{*}$ \\
\hline (6) & 3 & $5 \cdot 3 \pm 0.58$ & $4.5 \pm 0.67$ & $3.8 \pm 0.42$ \\
\hline Good $(>60)$ & 0 & $4 \cdot 3 \pm 0 \cdot 50$ & $4.2 \pm 0.69$ & $2 \cdot 9 \pm 0.31^{*}$ \\
\hline (5) & 3 & $5 \cdot 0 \pm 1 \cdot 0$ & $4.6 \pm 0.60$ & $3 \cdot 2 \pm 0.42$ \\
\hline All & 0 & $4 \cdot 4 \pm 0 \cdot 26$ & $3 \cdot 6 \pm 0 \cdot 25^{* *}$ & $2 \cdot 8 \pm 0 \cdot 15^{* * *}$ \\
\hline (28) & 3 & $4.7 \pm 0.82$ & $4 \cdot 1 \pm 0.28$ & $3 \cdot 1 \pm 0 \cdot 18^{* * *}$ \\
\hline
\end{tabular}

Data are means \pm SEM. $\dagger(\%$ motile after freezing and thawing $/ \%$ motile when fresh $) \times 100$ (before washing). Significant differences between fresh and GEYC-treated or between GEYCtreated and frozen-thawed spermatozoa after washing. ${ }^{* * *} P<0.001,{ }^{* *} P<0.01$, ${ }^{*} P<0.05$. The presence of significant variation was detected with a three-way analysis of variance and differences between individual cells of the table were tested subsequently by paired $t$ tests.

decrease in the percentage of progressively motile spermatozoa after mixing with GEYC was only poorly correlated $(r=0.298$ after elimination of two extreme outliers, which would otherwise have had a disproportionate influence on the result of the calculation) with the decrease after freezing and thawing. Different damage processes must therefore come into play during freezing or thawing and it is by protecting against these that the cryopreservative contributes to improved survival overall. Exposure to GEYC or freezing and thawing did not affect the ability of the spermatozoa to remain motile during incubation by contrast with the observations of Jeyendran et al. (1984). There are several differences in procedure that could explain this. Jeyendran et al. used only glycerol as opposed to glycerol-egg yolk-citrate and the semen was frozen in pellets on dry ice. There is no 
information to confirm that they diluted the thawed cryopreserved semen gradually to minimise osmotic stress on the spermatozoa. The ability of spermatozoa to maintain their motility can be an important indicator of their fertility (Irvine \& Aitken, 1986; Holt et al., 1989) but the $3 \mathrm{~h}$ incubation under favourable conditions used here may not have been sufficiently stressful to reveal any differences.

In our previous experiments (McLaughlin et al., 1990) we did not observe any effect of freezing and thawing on lateral head displacement. Although this could result from variation between the pools of donors used in the two experiments, there are technical factors that could explain the discrepancy. In the earlier experiments motility was measured by time lapse photography, whereas our measurements were made with a Hamilton Thorn analyser and different algorithms were used to determine head displacement. In the photographic method this was taken as the width of the track half-way along its length whereas in the Hamilton Thorn it is calculated as twice the maximum deviation of the centroid of the head from the average path. In this study measurements were made in a Makler chamber with a depth of $10 \mu \mathrm{m}$, whereas the photographic measurements were made on a slide with an average depth of $20 \mu \mathrm{m}$. Motility measurements can be affected by decreasing the chamber depth because of more frequent collisions between the spermatozoa and the glass (Ginsburg \& Amant, 1990). A decrease in lateral head displacement after cryopreservation was reported by Fernandes et al. (1990) but they did not wash the spermatozoa before making their motility measurements and their observations may have been affected by changes in viscosity.

The toxic effect of GEYC reported here is large, but toxic effects have been reported with concentrations of glycerol as low as $2 \%$ (Tulandi \& McInnes, 1984) and a substantial decrease in the percentage of motile spermatozoa in semen exposed to Human Sperm Preservation Medium containing $15 \%$ glycerol was observed by Critser et al. (1988). No decrease was found by Keel \& Webster (1989), who used $10 \%$ glycerol or $211 \mathrm{mmol}^{-1} \mathrm{~N}$-tris(hydroxymethyl) methyl-2aminoethane sulfonic acid $96 \mathrm{mmol} 1^{-1}$ hydroxymethylaminomethane (TEST)-buffered $20 \%$ egg yolk with $10 \%$ glycerol. However, their procedure differed in two important respects: observations were not made on matched samples from the same ejaculate subjected to each procedure and the motility measurements were made in the seminal plasma cryopreservative mixture thus avoiding the osmotic stress of rehydration. This suggests that the rapid expansion of cell volume that occurs upon dilution due to the rapid inflow of water compared with the slower outflow of glycerol is the major mechanism of damage. We were careful to add the diluent dropwise over 5 min to minimise this, but it may still be an important factor. Nevertheless other toxic effects of the constituents of GEYC cannot be ruled out. Glycerol can modify the structure of the plasma membrane and at high concentrations it can inhibit energy metabolism. Egg yolk may add phospholipids to the plasma membrane and thus modify its physical and permeability properties (see Hammerstedt et al., 1990). Egg yolk is a complex biological material and by virtue of its lipophilic nature could accumulate toxins ingested by the hen. Citrate is a potent feedback inhibitor of the key glycolytic enzyme, phosphofructokinase (Hoskins \& Stephens, 1968). A further possiblity is that the cells may have been damaged by the stress of centrifugation. Compressing the spermatozoa into a pellet renders them liable to damage by free oxygen radicals secreted by leucocytes or by defective spermatozoa (Aitken \& Clarkson, 1988). However, this should not be an important factor here, since none of the ejaculates had defective sperm function or a high round cell count when fresh.

There is a wide variation between the ability of semen from different men to survive cryopreservation (McLaughlin et al., 1992) and this increases the difficulty of recruiting sufficient donors to meet the demand for donor insemination. These data demonstrate that the variation between spermatozoa from different donors is not due to variation in their susceptibility to the toxic effects of cryopreservative but must reside in differences in sensitivity to the freezing process per se. Nevertheless the development of a less toxic cryopreservative could make a large contribution to the improvement of the quality of frozen human semen. One promising avenue is to decrease the concentration of glycerol in the medium and to substitute it with nonpenetrant molecules (Critser et al., 1988). Less glycerol would demand that faster freezing and thawing rates were used (see 
Watson, 1990) and this might require the introduction of more sophisticated equipment into DI clinics. The optimum glycerol concentration will also be influenced by the other constituents of the medium (Hammitt et al., 1988). The omission of glycerol without increases in the freezing rate or other changes in the medium results in a decrease in sperm motility (Jeyendran et al., 1984; Weidel \& Prins, 1987).

This work was supported by grant no. F1.87 from the Birthright Trust.

\section{References}

Aitken, R.J. (1983) The zona-free hamster egg penetration test. In Male Infertility, pp. 75-86. Ed. T. B Hargreave. Springer, Berlin.

Aitken, R.J. \& Clarkson, J.S. ( 1988) Significance of reactive oxygen species and antioxidants in defining the efficacy of sperm preparation techniques. Journal of Andrology 9, 367-376.

Critser, J.K., Huse-Benda, A.R., Aaker, D.V., Arneson, B.W. \& Ball, G.D. (1988) Cryopreservation of human spermatozoa. III. The effect of cryoprotectants on motility. Fertility and Sterility 50, 314-320.

Fernandes, P.A., McCoshen, J.A., Cheang, M., Kredentser, J.V. \& Wodzicki, A.M. (1990) Quantitative analysis of the effect of freezing on donor sperm motion kinetics. Fertility and Sterility 54, 322-327.

Ginsburg, K.A. \& Amant, D.R. (1990) The influence of chamber characteristics on the reliability of sperm concentration and movement measurements obtained by manual and videomicrographic analysis. Fertility and Sterility 53, 882--887.

Hammerstedt, R.H., Graham, K. \& Nolan, J.P. (1990) Cryopreservation of mammalian sperm: what we ask them to survive. Journal of Andrology 11, 73-88.

Hammitt, D.G., Walker, D.L. \& Williamson, R.A. (1988) Concentration of glycerol required for optimal survival and in vitro fertilizing capacity of frozen seprm is dependent on cryopreservation medium. Fertility and Sterility 49, 680-687.

Holt, W.V., Shenfield, F., Leonard, T., Hartman, T.D., North, R.D. \& Moore, H.D.M. (1989) The value of sperm swimming speed measurements in assessing the fertility of frozen semen. Human Reproduction 4, 292-297.

Hoskins, D.D. \& Stephens, D.T. (1968) Regulatory properties of primate sperm phosphofructokinase. Biochimica et Biophysica Acta 191, 292-302.

Hull, M.G.R., Glazener, C.M.A., Kelly, J., Conway, D.I., Foster, P.A., Hinton, R.A., Coulson, C., Lambert, P.A., Watt, E.M. \& Desai, K.M. (1985) Population study of causes, treatment and outcome of infertility. British Medical Journal 291, 1693-1697.
Irvine, D.S. \& Aitken, R.J. (1986) Predictive value of in vitro sperm function tests in the context of an AID service. Human Reproduction 1, 539--545.

Jeyendran, R.S., Van der Ven, H.H., Kennedy, W., PerezPelaez, M.E. \& Zaneveld, L.J.D. (1984) Comparison of glycerol and a Zwitterion buffer system as cryoprotective media for spermatozoa. Journal of Andrology 5, I-7.

Keel, B.A. \& Webster, B.W. (1989) Semen analysis data from fresh and cryopreserved donor ejaculates: comparison of cryoprotectants and pregnancy rates. Fertility and Sterility 52, 100-105.

McLaughlin, E.A., Ford, W.C.L. \& Hull, M.G.R. (1990) A comparison of the freezing of human semen in the uncirculated vapour above liquid nitrogen and in a commercial semi-programmable freezer. Human Reproduction 5, 724-728.

McLaughlin, E.A., Ford, W.C.L. \& Hull, M.G.R. (1992) The effect of cryopreservation on the motility characteristics and membrane integrity of human spermatozoa. Journal of Reproduction and Fertility 95, 527-534.

Mazur, P. (1984) Freezing of living cells: Mechanism and implications. American Journal of Physiology 247, C125-Cl42.

Tulandi, T. \& McInnes, R.A. (1984) Vaginal lubricants: Effects of glycerol and egg white on human sperm motility and progression in vitro. Fertility and Sterility 41, 151-153.

Watson, P.F. (1990) Artificial insemination and the preservation of semen. In Marshall's Physiology of Reproduction Vol. 2. Reproduction in the male, pp. 747-869. Ed. G. E. Lamming. Churchill Livingstone, Edinburgh.

Weidel, L. \& Prins, G.S. (1987) Cryosurvival of human spermatozoa frozen in eight different buffer systems. Journal of Andrology 8, 41-47. 